\title{
UTILIZAÇÃO DE HEMICELULOSES CATIÔNICAS, OBTIDAS A PARTIR DO APROVEITAMENTO DA PALHA DE MILHO, ASSOCIADAS COM TANINO PARA O TRATAMENTO DE EFLUENTES DE LAVANDERIA INDUSTRIAL
}

\author{
Elaine Angélica Mundim Ribeiro ${ }^{a}$, Fúlvio Rafael Bento de Souza ${ }^{a}$, Fábio Augusto do Amarala , Guimes Rodrigues Filhoa,*,\#, \\ Raquel Maria Ferreira de Sousa ${ }^{a}$, Júlia Graciele Vieira ${ }^{a}$, Talita Ferreira de Rezende Costa ${ }^{a}$, José Pedro Thompson Juniora \\ Rosana Maria Nascimento de Assunção ${ }^{\mathrm{b}}$ e Sheila Cristina Canobre ${ }^{\mathrm{a}}$ \\ anstituto de Química, Universidade Federal de Uberlândia, Campus Santa Mônica, 38.400-902, Uberlândia - MG, Brasil \\ ${ }^{b}$ Faculdade de Ciências Integradas do Pontal, Universidade Federal de Uberlândia, Campus do Pontal, 38302-000, Ituiutaba - MG, \\ Brasil
}

Recebido em 21/02/2016; aceito em 12/07/2016; publicado na web em 19/08/2016

\begin{abstract}
UTILIZATION OF CATIONIC HEMICELLULOSES, OBTAINED FROM THE CORN HUSK, IN ASSOCIATION WITH TANNIN FOR USE IN WASTEWATER TREATMENT OF INDUSTRIAL LAUNDRY. The synthesis of cationic derivatives of hemicelluloses from corn husk was carried out by the reaction of hemicelluloses with 2,3-epoxypropyltrimethylammonium chloride (ETA) in aqueous sodium hydroxide. The characterization of the cationic derivatives was carried out by elemental analysis, Fourier Transformed Infrared Spectroscopy (FTIR) and Thermogravimetric Analysis (TG/DTA). An important characteristic was the solubility in water of the produced derivatives. This characteristic was used to apply derivatives as auxiliary coagulation with tannin in wastewater treatment for industrial laundries. The results showed that the association of tannin and cationic hemicelluloses $\left(3,200 \mathrm{mg} \mathrm{L}^{-1}\right)$ is important because the turbidity removal was greater than $95 \%$ in all range of $\mathrm{pH}$, including alkaline regions. Then it is unnecessary to adjust the $\mathrm{pH}$ before the coagulation step.
\end{abstract}

Keywords: cationic hemicelluloses; corn husk; wastewater treatment; industrial laundries.

\section{INTRODUÇÃO}

Atualmente o aproveitamento de recursos renováveis tem se tornado imprescindível na política mundial de sustentabilidade. Neste contexto, resíduos agroindustriais como bagaço de cana-de-açúcar, sabugo e palha de milho têm sido estudados para produzir energia através da sua queima, produzir bioetanol e obter produtos químicos derivados.

Os resíduos agroindustriais são ricos em celulose e hemicelulose, que apresentam, em sua estrutura, hidroxilas que podem sofrer uma série de reações tais como esterificação, eterificação, etc. Na literatura alguns dos principais derivados produzidos com a hemicelulose ou com a celulose são: i) derivados metilados; ${ }^{1,2}$ ii) derivados acetilados e sulfonados ${ }^{3,4}$ e iii) derivados catiônicos..$^{5-8}$

As hemiceluloses são copolímeros amorfos de polissacarídeos heterogêneos, com um grau de polimerização que varia entre 80 e 200. Ao contrário da celulose, as hemiceluloses têm uma combinação aleatória de monossacarídeos incluindo principalmente pentoses ( $\beta$-D-xilose, $\beta$-L-arabinose), hexoses ( $\beta$-D-glicose, $\beta$-D-manose, $\beta$-D-galactose) e ácidos urônicos. ${ }^{9}$ Esse copolímero natural é o segundo mais abundante do reino vegetal depois da celulose e a sua quantidade varia de acordo com a espécie de planta. ${ }^{10}$ Por exemplo, o bagaço de cana é constituído de $25-34 \%$ de hemiceluloses, ${ }^{11}$ a palha do trigo possui uma quantidade de hemiceluloses que varia entre $32-38 \%{ }^{10}$ e a palha de milho apresenta quantidade desse copolímero natural que corresponde a cerca de $35 \% .{ }^{12}$ Dentre os componentes das hemiceluloses, as xilanas são as mais abundantes em gramíneas. ${ }^{12} \mathrm{As}$ xilanas são formadas por unidades de $\beta$-D-xilose na cadeia principal e diferentes carboidratos, tais como ácidos urônicos e seus derivados, L-arabinose e oligossacarídeos em cadeias laterais. ${ }^{13,14}$

*e-mail: guimes.rodriguesfilho@gmail.com

"e-mail alternativo: guimes@ufu.br
A hemicelulose apresenta um grande potencial químico reacional devido à presença de cadeias laterais em sua estrutura, característica essa que confere à mesma uma conformação espacial menos impedida para reações químicas nas hidroxilas das unidades de $\beta$-D-xilose. ${ }^{10}$ Recentemente, Landim et al. ${ }^{8}$ estudaram a utilização de derivados catiônicos da hemicelulose no tratamento de esgoto das estações da cidade de Uberlândia-MG.

O Departamento de Agricultura dos Estados Unidos (USDA) projetou para a produção mundial de milho 2013/2014 uma safra de 966,9 milhões de toneladas. Para o Brasil o Ministério da Agricultura, Pecuária e Abastecimento projetou para 2013/2014 uma safra entre 78,8 e 89,0 milhões de toneladas e para 2022/2023 a projeção é de 96,0 milhões de toneladas, sendo que o Estado de Minas Gerais, onde se situa o nosso grupo de pesquisa, responde por $9,5 \%$ da produção. Juntamente com essa produção, são geradas grandes quantidades de palha e sabugo de milho, que são resíduos ricos em celulose e hemicelulose. Aliando estes fatos ao desenvolvimento de novos materiais utilizando matéria-prima renovável, neste trabalho é descrita a preparação de derivados catiônicos de hemicelulose a partir da palha de milho, visto que derivados catiônicos de celulose e amido já se mostraram promissores em diversas aplicações. ${ }^{7,15-17}$

Assim, a rota de síntese da hemicelulose cationica baseou-se no trabalho de Ren et al. ${ }^{6}$ que prepararam derivados catiônicos de hemicelulose de bagaço de cana-de-açúcar usando como reagente quaternizante o cloreto de 2,3-epoxipropiltrimetilamônio (ETA). Para a caracterização dos derivados catiônicos de hemicelulose obtidos da palha de milho empregou-se a análise elementar, análise termogravimétrica (TGA) e espectroscopia vibracional de absorção no infravermelho (FT-IR).

O derivado catiônico foi aplicado como auxiliar de coagulação do coagulante tanino, também de fonte renovável, em ensaios de coagulação para o tratamento de efluentes provenientes de uma lavanderia industrial. Na literatura há alguns trabalhos aplicando o tanino 
como coagulante, mas não há aplicando-o associado à hemicelulose catiônica. Ademais, os resultados de coagulação mostram a ação do tanino em faixa restrita de $\mathrm{pH} .{ }^{18-20}$

Assim, a premissa do presente trabalho foi de que o derivado catiônico das hemiceluloses oferecesse um mecanismo de coagulação diferente do tanino e, portanto, pudesse atuar como auxiliar de coagulação no tratamento de efluente provindo de uma empresa de lavanderia industrial. Para isso, o objetivo foi aplicá-lo em ação associada ao tanino, visando aumento da coagulação no tratamento físico-químico do mesmo. Os parâmetros avaliados foram: $\mathrm{pH}$, a DQO, condutividade e remoção da turbidez. De acordo com Ren et al., ${ }^{21,22}$ os derivados hemicelulósicos, geralmente, são mais solúveis quando comparados aos derivados da celulose, e este é um ponto diferencial para o tratamento de águas residuárias.

\section{PARTE EXPERIMENTAL}

\section{Materiais}

A palha de milho utilizada na obtenção da hemicelulose foi previamente lavada com água destilada, seca a $50{ }^{\circ} \mathrm{C}$ por 24 horas e armazenada em temperatura ambiente. Os reagentes hidróxido de potássio, hidróxido de sódio, etanol 98\%, ácido acético glacial, ácido clorídrico e dimetilsulfóxido foram adquiridos da empresa Vetec e o cloreto de 2,3-epoxipropiltrimetilamônio (ETA) e o clorito de sódio foram adquiridos da empresa Sigma-Aldrich. O Tanino Tanfloc SL, com viscosidade média acima de 50 segundos medidos em copo Ford número 4, $\mathrm{pH}$ do xarope variando entre 1,32,5, sólidos totais entre $23 \%-37 \%$, foi obtido da empresa TANAC S. A. Todos os reagentes são de grau analítico e foram utilizados sem purificação adicional. Os efluentes foram provenientes de uma Lavanderia Industrial com unidades em Belo Horizonte-Minas Gerais e Arujá-São Paulo.

\section{Obtenção da hemicelulose}

$\mathrm{O}$ isolamento da hemicelulose $\mathrm{A}$ foi realizado de acordo com o procedimento de Vieira et al. ${ }^{1}$

\section{Síntese do derivado de hemicelulose catiônica}

Para a obtenção dos derivados catiônicos foi realizado o procedimento a seguir, o qual foi adaptado de Ren et $a l .{ }^{6} \mathrm{Em} 0,5 \mathrm{~g}$ de hemicelulose $\left(0,0076 \mathrm{~mol}\right.$ de grupo $\left.\mathrm{OH}^{-}\right)$foram adicionados em $5 \mathrm{~mL}$ de água destilada e deixou-se agitando a $60{ }^{\circ} \mathrm{C}$ por $30 \mathrm{~min}$. Adicionou-se $0,7 \mathrm{~g}$ de $\mathrm{NaOH}$ e deixou-se agitando por $20 \mathrm{~min}$. A seguir, adicionou-se o ETA na proporção estabelecida de acordo com o número de grupos $\mathrm{OH}$, ou seja, para o derivado 1 razão molar ETA/ $\mathrm{OH}$ das xilanas igual a 3 e o derivado 2 razão molar ETA/OH das xilanas igual a 6 , deixou-se agitando por $30 \mathrm{~min}$, adicionou-se 1,1 g de $\mathrm{NaOH}$ e manteve-se o meio reacional sob agitação a $60^{\circ} \mathrm{C}$ por 5 horas. Ao final, o meio reacional foi resfriado, neutralizado com $\mathrm{HCl}$ e a hemicelulose catiônica foi precipitada com etanol $98 \%$ e filtrada em funil de placa porosa número 4 . Os derivados 1 e 2 foram dissolvidos em água e reprecipitados com etanol $98 \%$.

\section{Determinação do rendimento e do grau de substituição (GS)}

O cálculo do grau de substituição (GS) foi realizado através da quantidade de nitrogênio e carbono que o derivado catiônico da hemicelulose possui, de acordo com a equação $1:^{21}$

$$
\mathrm{GS}=(60 \times \% \mathrm{~N}) /(14 \times \% \mathrm{C}-72 \times \% \mathrm{~N})
$$

Para o cálculo do rendimento foi considerado que quando um GS igual a 2,0 for obtido, o rendimento será de $100 \%$, e para um GS de 0,0 o rendimento será de $30,3 \% .^{22}$

Os dados da análise elementar utilizados para a determinação do GS dos derivados catiônicos 1 e 2 são mostrados na Tabela 1 .

Tabela 1. Dados da análise elementar das hemiceluloses catiônicas 1 e 2

\begin{tabular}{cccc}
\hline $\begin{array}{c}\text { Hemiceluloses } \\
\text { catiônicas }\end{array}$ & $\mathrm{C} / \%$ & $\mathrm{H} / \%$ & $\mathrm{~N} / \%$ \\
\hline \multirow{2}{*}{1} & 9,78 & 1,87 & 0,74 \\
2 & 9,08 & 1,60 & 0,67 \\
\hline \multirow{2}{*}{2} & 5,41 & 0,82 & 0,32 \\
& 5,44 & 0,62 & 0,26 \\
\hline
\end{tabular}

\section{Caracterização dos derivados catiônicos da hemicelulose}

A espectroscopia vibracional de absorção no infravermelho da hemicelulose natural e dos seus derivados foi realizada utilizando o equipamento Shimadzu IR Prestige-21. As pastilhas foram preparadas com $\operatorname{KBr}(1 / 100(\mathrm{~m} / \mathrm{m}))$.

As análises termogravimétricas (TG/DTA) foram realizadas em um equipamento TGA-50, Shimadzu. A massa de amostra utilizada foi de $10 \mathrm{mg}$. As amostras foram aquecidas da temperatura ambiente até $900{ }^{\circ} \mathrm{C}$ a uma taxa de $20^{\circ} \mathrm{C}$ min $^{-1}$ em atmosfera de nitrogênio de fluxo de $50 \mathrm{~mL} \mathrm{~min}^{-1}$.

As análises elementares foram realizadas em um aparelho Perkin Elmer, Elemental Analyser modelo $2400 \mathrm{CHN}$, sendo a temperatura de combustão do material de $925^{\circ} \mathrm{C}$, em presença de oxigênio puro na Central Analítica da Universidade de São Paulo-SP. Os gases produzidos durante a combustão foram separados pela coluna e detectados por detector de condutividade térmica.

\section{Aplicação das hemiceluloses catiônicas como auxiliar de coagulação}

As hemiceluloses catiônicas-1 foram avaliadas como auxiliadoras de coagulação do coagulante tanino, também de fonte renovável, em ensaios de coagulação. As amostragens foram realizadas de maneira composta com coletas diárias de 3 em 3 horas durante 7 dias, a fim de se obter uma composição de efluente bruto similar ao encontrado no tanque de recebimento da estação local. Para o tratamento físico-químico do efluente foram realizadas coletas de $0,875 \mathrm{~L}$ a cada 3 horas diretamente do tanque de equalização da estação de tratamento de efluentes (ETE) por 7 dias consecutivos, totalizando $49 \mathrm{~L}$ de efluente por semana. A utilização do coagulante tanino e dos derivados de hemicelulose como auxiliares de coagulação foram realizadas em um aparelho de "Jar Test" (Nova Ética, modelo 218) com 6 jarros providos de sistema ajustável de agitação.

\section{Construção dos diagramas de coagulação}

O tratamento do efluente da lavanderia industrial foi realizado com o uso das hemiceluloses catiônicas-1 como auxiliar de coagulação após o uso do tanino classe SL como coagulante primário. Desse modo, um diagrama de coagulação foi construído para obtenção de uma remoção de turbidez máxima com dosagens mínimas do coagulante primário e do auxiliar de coagulação. Este diagrama será apresentado separadamente na forma bidimensional pela variação de remoção de turbidez em função do pH de aplicação. Para a construção do diagrama de coagulação, inicialmente o efluente foi acidificado com ácido sulfúrico até $\mathrm{pH} 2$, para retirada de óleos e graxas e, em 
seguida, filtrado. Nesta etapa, quantidades fixas de efluente $(200 \mathrm{~mL})$ tiveram seus $\mathrm{pH}$ ajustados numa faixa de 3 a 10 .

\section{Investigação das hemiceluloses catiônicas-1 como floculante}

Para verificar se as hemiceluloses poderiam atuar como floculantes, a mesma metodologia foi realizada em tratamento com tanino classe SL, substituindo-se a PAC (poliacrilamida catiônica - floculante sintético) por hemiceluloses catiônicas, aplicadas nas mesmas condições. Para isto, uma dosagem de $250 \mathrm{mg} \mathrm{L}^{-1}$ de tanino (baixa dosagem) foi aplicada para uma concentração fixa de $8 \mathrm{mg} \mathrm{L}^{-1}$ de hemiceluloses catiônicas.

\section{Investigação das hemiceluloses catiônicas-1como auxiliar de coagulação}

O coagulante tanino classe SL foi aplicado numa dosagem de $3000 \mathrm{mg} \mathrm{L}^{-1}$ (dosagem intermediária) para cada $\mathrm{pH}$ ajustado. Posteriormente, as hemiceluloses cationicas-1 foram adicionadas numa dosagem de $8 \mathrm{mg} \mathrm{L}^{-1}$, ambos sendo adicionados com uma agitação sob gradiente de velocidade de $100 \mathrm{~s}^{-1}$. As medidas do índice de turbidez do efluente foram realizadas em três etapas distintas, antes e após adição do coagulante (tanino classe SL) e, por fim, após adição do auxiliar de coagulação (hemiceluloses catiônicas-1).

Posteriormente, a dosagem de tanino foi fixada em $3000 \mathrm{mg} \mathrm{L}^{-1}$ (de efetiva atuação quando aplicado sem posterior adição do polieletrólito PAC), aumentando-se as dosagens de hemiceluloses catiônicas-1 gradativamente (de $200 \mathrm{mg} \mathrm{L}^{-1}$ a $3200 \mathrm{mg} \mathrm{L}^{-1}$ ) a fim de encontrar as dosagens apropriadas do coagulante e do auxiliar de coagulação. O diagrama de coagulação para a associação de tanino classe SL com hemiceluloses catiônicas-1 é mostrado no fluxograma da Figura 1.

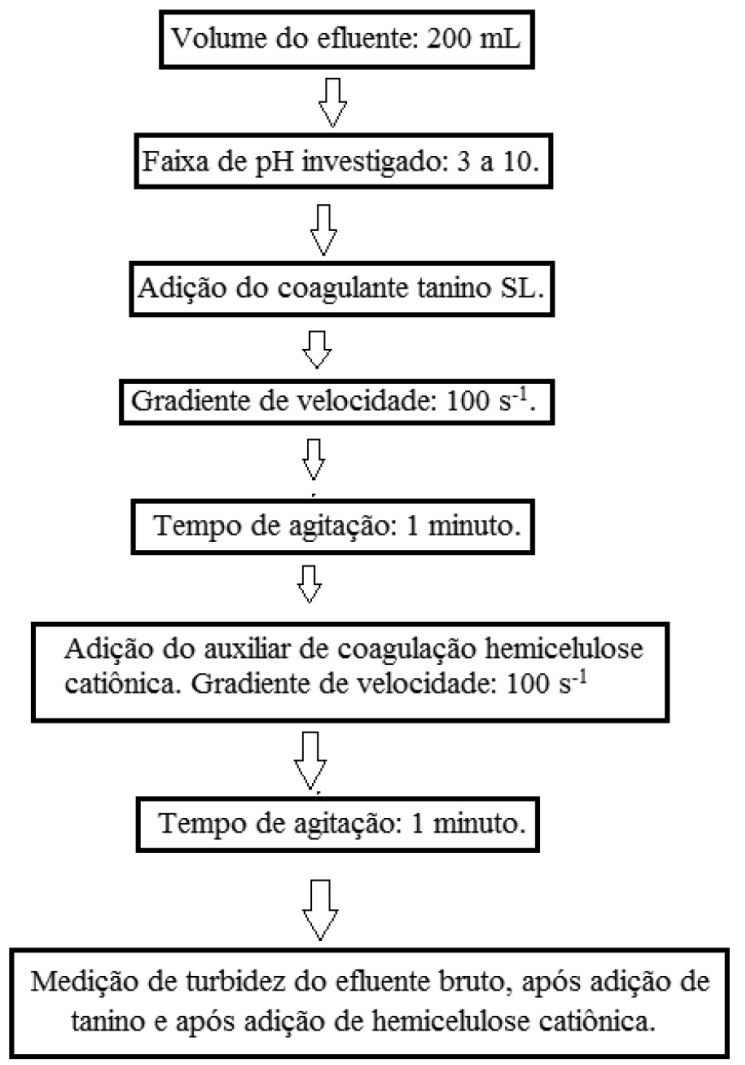

Figura 1. Fluxograma esquemático da construção dos diagramas de coagulação

\section{Tratamento físico químico do efluente}

O tratamento físico-químico do efluente bruto foi estabelecido pelas seguintes etapas: abaixamento de $\mathrm{pH}$ para retirada de óleos e graxas, equalização do efluente até $\mathrm{pH}$ básico, aplicação dos coagulantes/auxiliares/floculantes e neutralização.

\section{RESULTADOS E DISCUSSÃO}

\section{Determinação do rendimento e grau de substituição}

A reação de eterificação da hemicelulose $\mathrm{A}$ da palha de milho em meio básico utilizando ETA comercial como agente cationizante ocorre segundo o mecanismo proposto na Figura 2, no qual o oxigênio dos grupos hidroxilas da hemicelulose ataca o carbono menos impedido do epóxido (ETA) dando origem ao composto eterificado.

Na Tabela 2 se encontram os rendimentos da reação, grau de substituição, bem como a solubilidade em água dos derivados obtidos.

De acordo com Gaspár et al. ${ }^{21}$ a xilana é o principal tipo de açúcar presente na hemicelulose da palha de milho, portanto, predominam unidades de $\beta$-D-xilose. Isto faz com que a hemicelulose da palha de milho contenha, predominantemente, duas hidroxilas por unidade de $\beta$-D-xilose disponíveis para serem eterificadas. Deste modo, de acordo com Gaspar et al., ${ }^{21}$ para o cálculo da razão molar, considera-se que a hemicelulose seja constituída apenas de xilose (MM=132).

O aumento da concentração de ETA não resultou em maior grau de substituição. Isso ocorreu porque, como se manteve a concentração de $\mathrm{NaOH}$ constante, não havia mais oxigênios desprotonados de grupos glicosídicos para reagir. Além disso, o excesso de $\mathrm{NaOH}$ provocou a hidrólise do epóxido do ETA, reduzindo o GS. Neste caso, seria necessário aumentar também a proporção ETA/NaOH. Entretanto, isso não foi realizado, pois, segundo Ren et al. ${ }^{6} \mathrm{o}$ aumento desta proporção eleva a clivagem das ligações glicosídicas, reduzindo o tamanho da cadeia polimérica.

Ambos os derivados, ao contrário da hemicelulose natural, apresentaram-se solúveis em água, sendo que as hemiceluloses catiônicas-1 apresentaram maior solubilidade que as hemiceluloses catiônicas-2, provavelmente devido à maior presença de grupos catiônicos, comprovado pelo maior grau de substituição observado.

\section{Caracterização por espectroscopia no infravermelho}

A Figura 3 apresenta o espectro na região do infravermelho da hemicelulose natural ( $\mathrm{NH}$ ) e dos derivados catiônicos $\mathbf{1}$ e 2. Observase na Figura 3a que tanto a hemicelulose natural como as catiônicas possuem bandas características nas regiões: $3421 \mathrm{~cm}^{-1}$ devido ao estiramento das ligações O-H, $2920 \mathrm{~cm}^{-1}$ característico de estiramento de ligações $\mathrm{C}$-H de grupos $\mathrm{CH}_{2} \mathrm{e} \mathrm{CH}_{3}, 1635 \mathrm{~cm}^{-1}$ deformação angular de ligação H-O-H da água absorvida pela amostra, $1475 \mathrm{~cm}^{-1}$ relacionadas à deformação assimétrica de $\mathrm{CH}_{3}, 1419 \mathrm{~cm}^{-1}$ estiramento de $\mathrm{C}-\mathrm{N}$ de amina quaternária, $1254 \mathrm{~cm}^{-1}$ referente ao estiramento de ligação C-O-C de grupos etílicos, $1049 \mathrm{~cm}^{-1}$ devido ao estiramento das ligações C-O de alcoóis primários e $908 \mathrm{~cm}^{-1}$ estiramento característico das ligações $\beta$-glicosídicas entre as unidades de açúcar existentes nas hemiceluloses. A Tabela 3 mostra as principais atribuições das bandas.

Analisando a Figura $3 \mathrm{~b}$ pode-se observar que, de acordo com Ren et al., ${ }^{21,22}$ nos espectros dos derivados catiônicos 1 e 2 há o surgimento de uma banda na região de $1483-1467 \mathrm{~cm}^{-1}$ referente à deformação angular assimétrica de grupos $\mathrm{CH}_{3}$ oriundos do grupo substituinte catiônico. Esta banda está ausente no espectro da hemicelulose natural, o que corrobora a efetividade da reação de substituição demonstrada pelos valores de GS e pela solubilidade em água dos derivados. 


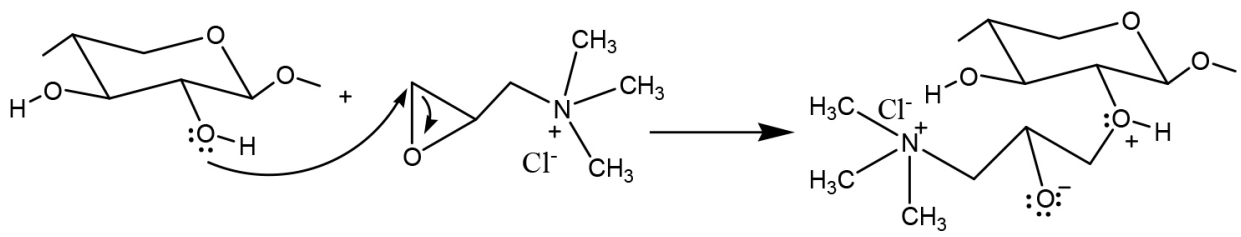

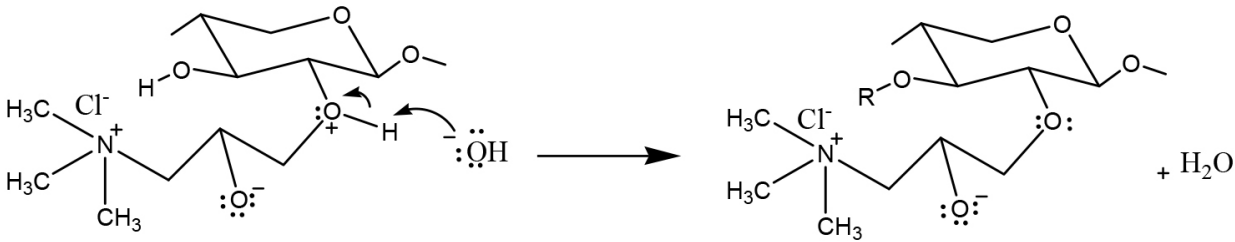

$\mathrm{R}=\mathrm{CH}_{2} \mathrm{CHOHCH}_{2} \mathrm{~N}^{+}\left(\mathrm{CH}_{3}\right)_{3}$

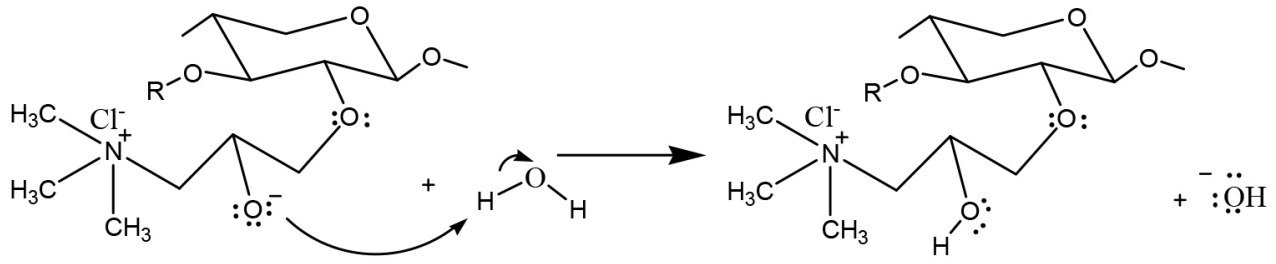

Figura 2. Mecanismo de quaternização de hemiceluloses

Tabela 2. Valores de GS, rendimento da derivatização e solubilidade em água

\begin{tabular}{ccccc}
\hline $\begin{array}{c}\text { Derivado } \\
\text { catiônico }\end{array}$ & $\begin{array}{c}\text { Razão molar } \\
\text { de ETA/OH }\end{array}$ & GS & $\begin{array}{c}\text { Rendimento } \\
(\%)\end{array}$ & $\begin{array}{c}\text { Solubilidade } \\
\left(\mathrm{g} \mathrm{L}^{-1}\right)\end{array}$ \\
\hline 1 & 3 & 0,52 & 48,44 & 20,0 \\
2 & 6 & 0,32 & 41,31 & 3,2 \\
\hline
\end{tabular}

Tabela 3. Descrição das principais atribuições das bandas dos espectros de FTIR

\begin{tabular}{|c|c|c|c|}
\hline \multicolumn{3}{|c|}{ Comprimento de onda $\left(\mathrm{cm}^{1}\right)$} & \multirow{2}{*}{ Atribuições } \\
\hline $\mathbf{H N}$ & 1 & 2 & \\
\hline 3421 & 3421 & 3421 & estiramento de $\mathrm{O}-\mathrm{H}$ \\
\hline 2920 & 2920 & 2928 & estiramento de $\mathrm{C}-\mathrm{H}$ dos grupos $\mathrm{CH}_{2}$ e $\mathrm{CH}_{3}$ \\
\hline 1635 & 1635 & 1636 & $\begin{array}{l}\text { deformação angular de H-O-H de água } \\
\text { absorvida }\end{array}$ \\
\hline- & 1483 & 1483 & deformação angular assimétrica de $\mathrm{CH}_{3}$ \\
\hline- & 1419 & 1419 & estiramento de $\mathrm{C}-\mathrm{N}$ de amina quaternária \\
\hline 1254 & 1254 & 1254 & estiramento de $\mathrm{C}-\mathrm{O}-\mathrm{C}$ de éter \\
\hline 1049 & 1049 & 1049 & estiramento C-O de alcoóis primários \\
\hline 908 & 908 & 908 & ligações $\beta$-glicosídicas \\
\hline
\end{tabular}

Ainda que os espectros de FTIR sejam aqui utilizados apenas de forma qualitativa, as relações das alturas das bandas de estiramento $\mathrm{CH}_{2}, \mathrm{CH}_{3}$ para as bandas de estiramento $\mathrm{OH}\left(v\left(\mathrm{CH}_{2}, \mathrm{CH}_{3}\right) / v(\mathrm{OH})\right)$ estão aproximadamente nas seguintes razões: hemicelulose natural $(0,14)$; hemicelulose catiônica $2(0,25)$; hemicelulose catiônica 1 $(0,40)$, ou seja, praticamente na mesma razão dos GS para os derivados catiônicos.

\section{Análise termogravimétrica (TG/DTA)}

As Figuras 4 e 5 apresentam os termogramas das análises térmicas de TGA/DTA e de DTG, respectivamente, para a hemicelulose natural e seus derivados catiônicos 1 e 2 .
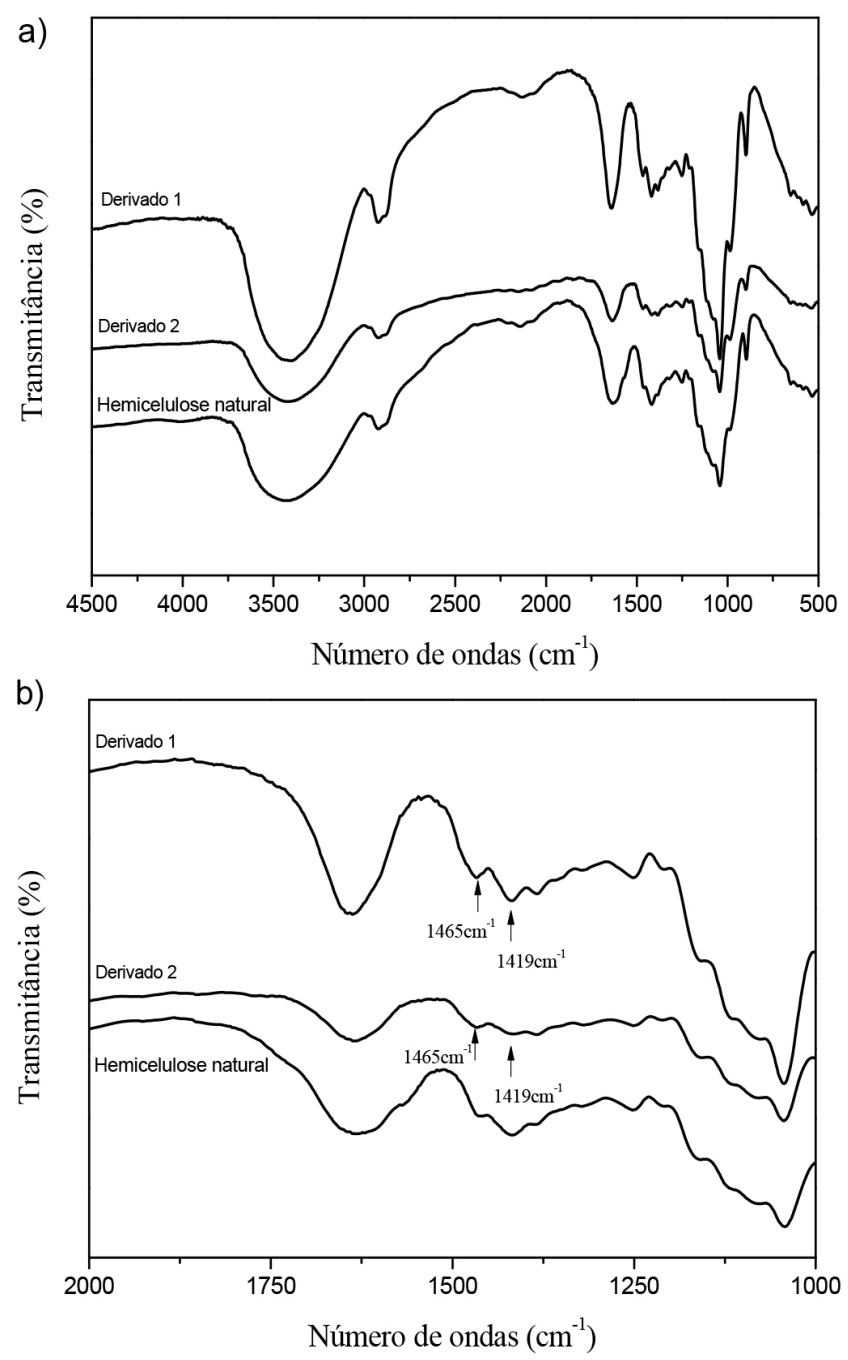

Figura 3. Espectros de FTIR da hemicelulose natural e dos derivados catiônicos 1e 2: a) 4500 a $500 \mathrm{~cm}^{-1}$; e b) expansão da região de 2000 a $1000 \mathrm{~cm}^{-1}$ 

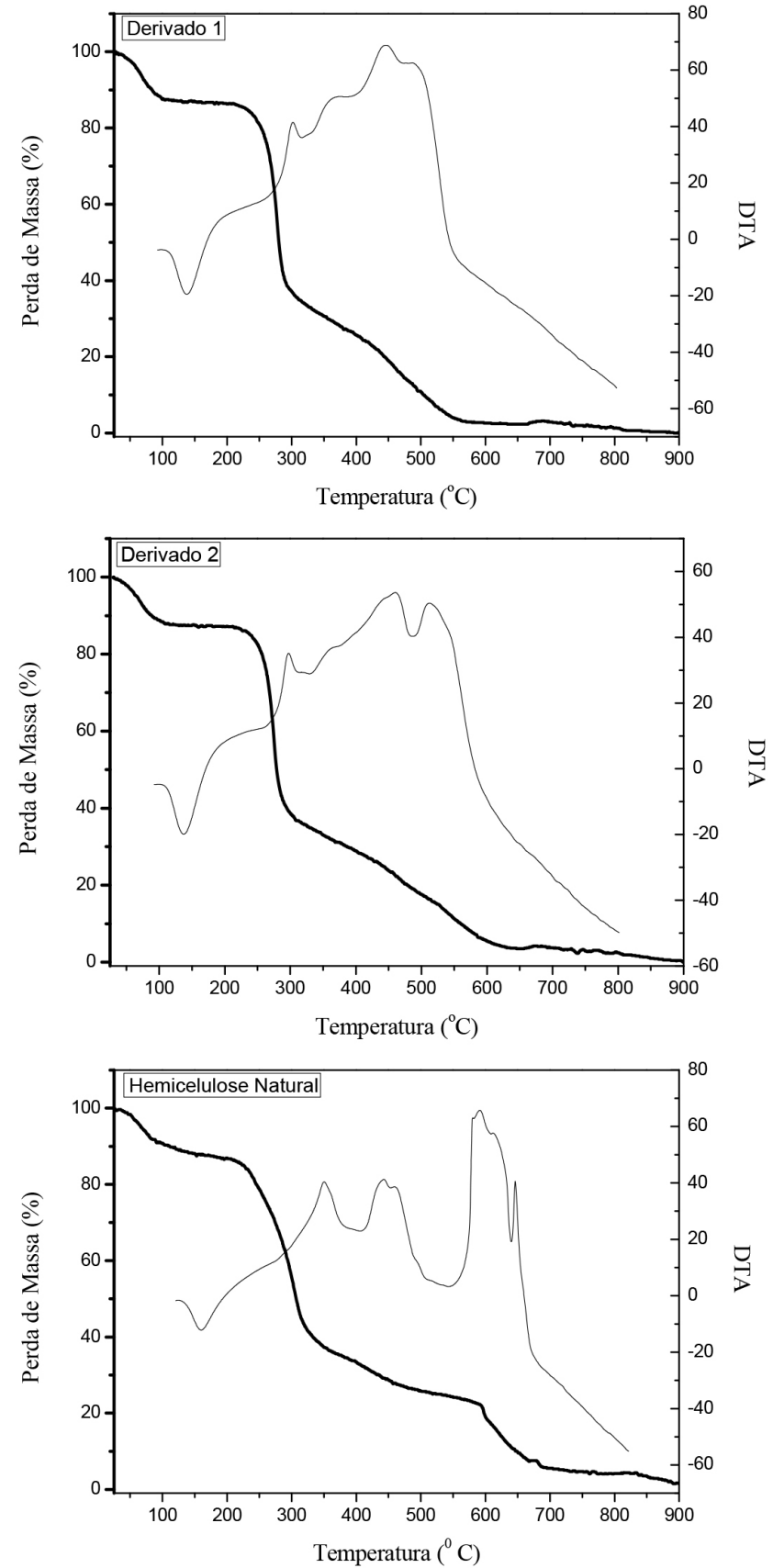

Figura 4. Termogramas TG/DTA da hemicelulose natural e dos derivados catiônicos 1 e 2

Na Figura 4 podemos observar nas curvas de DTA as seguintes características para todas as amostras: pico endotérmico, que corresponde à saída de água localizado entre 100 e $200{ }^{\circ} \mathrm{C}$; picos exotérmicos na região de 300 a $700{ }^{\circ} \mathrm{C}$, os quais podem ser atribuídos ao rompimento das interações intermoleculares e à decomposição do polímero. Observa-se também que todos os picos encontrados nas curvas de DTA se encontram em temperaturas mais elevadas para a hemicelulose natural. Isto deve ser atribuído ao fato de que a derivatização rompe as ligações de hidrogênio sendo que os materiais produzidos tornam-se assim menos estáveis termicamente do que a hemicelulose natural.

Quando analisamos os resultados das curvas de DTA, Figura 4, observamos que a principal perda de massa, cerca de $50 \%$, tem característica exotérmica e corresponde à decomposição da hemicelulose através de uma série de reações químicas. ${ }^{23} \mathrm{Na}$ hemicelulose natural esta decomposição ocorre em dois estágios. O primeiro estágio é representado por um ombro, localizado em aproximadamente $250{ }^{\circ} \mathrm{C}$, o qual é atribuído principalmente à clivagem das ligações glicosídicas e a decomposição da estrutura da cadeia lateral (tais como a unidade de ácido 4-O-metilglucurônico), enquanto que o segundo estágio é representado por um pico, localizado em aproximadamente $300{ }^{\circ} \mathrm{C}$, e deve ser atribuído à fragmentação de outras unidades despolimerizadas (unidades xilanas). ${ }^{23}$

No caso dos derivados catiônicos 1 e 2 observa-se nas curvas DTG, Figura 5, apenas um pico localizado em ambas as amostras em aproximadamente $275{ }^{\circ} \mathrm{C}$. A localização deste pico em uma temperatura entre as duas citadas anteriormente para a hemicelulose natural significa que nos derivados catiônicos os fenômenos de
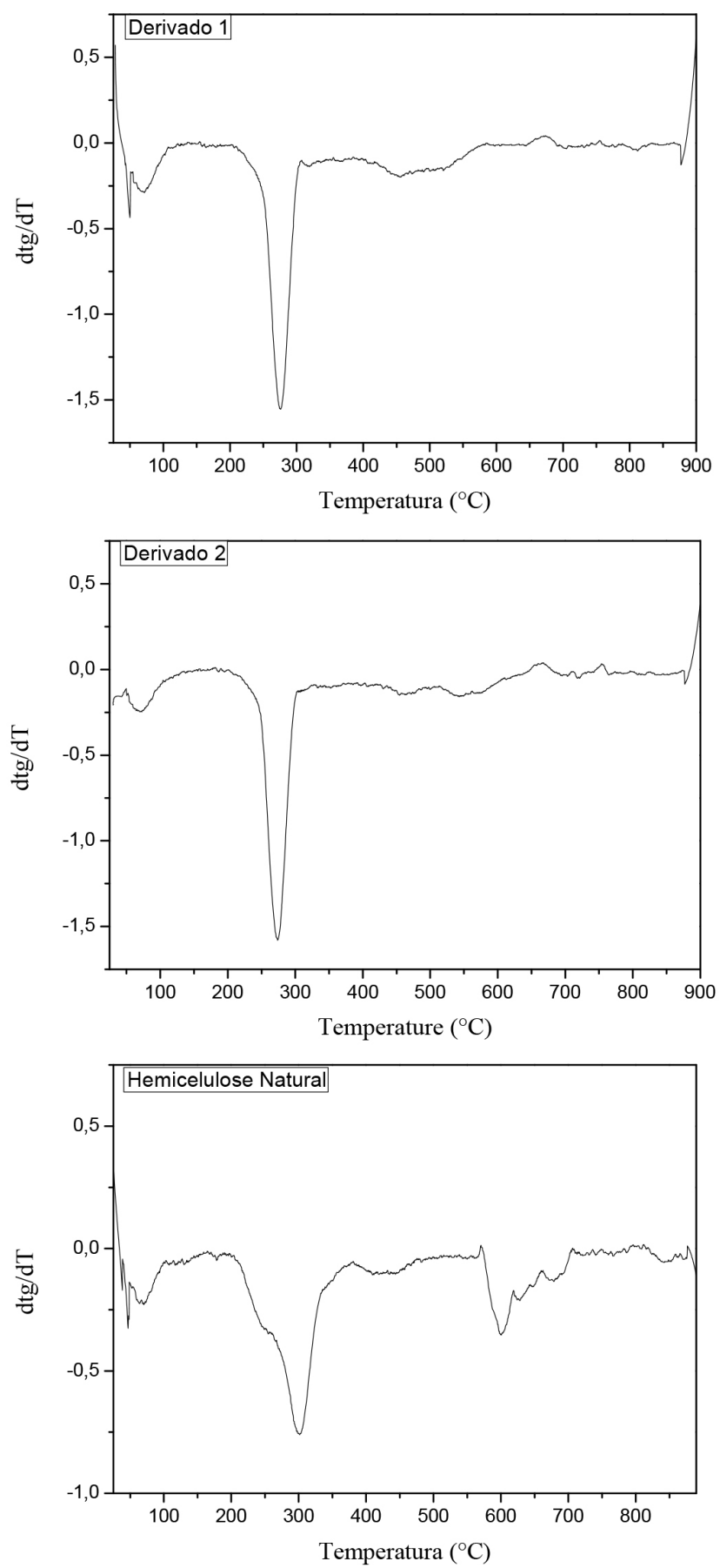

Figura 5. Curvas DTG da hemicelulose natural e dos derivados catiônicos 1 e 2 
despolimerização das unidades de xilanas e de decomposição de cadeias laterais ocorrem simultaneamente, corroborando assim a eficiência da síntese dos derivados pela rota empregada neste trabalho. Além disso, a localização dos picos indica que os derivados catiônicos são menos estáveis termicamente do que a hemicelulose natural. No entanto, a faixa de temperatura utilizada no tratamento do efluente não influenciará na estabilidade térmica desses derivados catiônicos.

\section{Investigação das hemiceluloses catiônicas-1 como floculante}

Martinez ${ }^{24}$ citado por Coral et al. ${ }^{25}$ aponta que o tanino (Tanfloc $S L$ ) atua como coagulante, neutralizando cargas e formando pontes entre as partículas coloidais, não consumindo a alcalinidade do meio, sendo efetivo somente em uma faixa de $\mathrm{pH}$ de 4,5 a 8,0. As hemiceluloses catiônicas-1, quando utilizadas como coagulante primário, não apresentaram remoções de turbidez satisfatórias, visto a característica acentuada de turbidez e sólidos suspensos do efluente em questão. Desta forma, investigou-se sua aplicação como floculante, em substituição à poliacrilamida, já utilizada para o processo de floculação do efluente em questão.

A Figura 6 mostra a variação da remoção de turbidez em função do $\mathrm{pH}$ para a aplicação do tanino como coagulante primário, associado à poliacrilamida ou à hemicelulose catiônica, como floculantes.

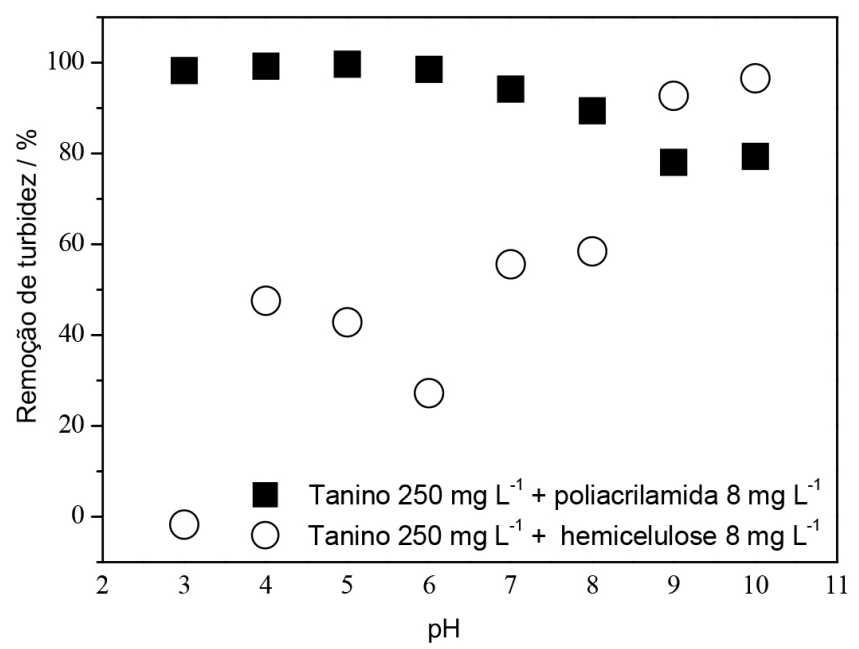

Figura 6. Variação da remoção de turbidez em função do $\mathrm{pH}$ para aplicação de tanino (coagulante primário) e poliacrilamida ou hemicelulose catiônica (floculantes) no tratamento de efluente de lavanderia industrial

A aplicação do tanino associado à poliacrilamida forneceu valores próximos a $100 \%$ de remoção de turbidez em pHs ácidos e próximo a região do $\mathrm{pH}$ neutro, sendo reduzidos para aproximadamente $80 \%$ na faixa básica. Nesta faixa de dosagem, o processo de coagulação do tanino acontece principalmente pelo mecanismo de arraste em que as interações ocorrem em maior quantidade e envolvem todas as partículas que participam do sistema em suspensão do efluente. ${ }^{26} \mathrm{~A}$ poliacrilamida, um polímero de elevada massa molecular, atua como floculante, densificando os coágulos formados do coagulante tanino com consequente sedimentação. Cabe ressaltar uma ligeira redução nas remoções de turbidez para a faixa básica de $\mathrm{pH}$, considerando a baixa dosagem de coagulante utilizada para tal efluente contendo elevada quantidade de sólidos suspensos e sedimentáveis. ${ }^{27}$ Desta forma, sugere-se que o aumento do $\mathrm{pH}$ inibe o predomínio de cargas do coagulante, resultando em uma menor remoção dos sólidos suspensos e da turbidez. ${ }^{28}$

Já para a associação do tanino às hemiceluloses catiônicas-1 (aplicada na mesma dosagem do PAC) valores de remoção de turbidez inferiores a $60 \%$ foram observados para $\mathrm{pHs}$ ácidos e neutro, com crescente melhoria da remoção de turbidez em pHs acima de 8 , na qual a ação coagulante do tanino é desfavorecida. ${ }^{29}$ No entanto, considerou-se que tais dosagens aplicadas foram insuficientes para que o processo de coagulação ocorresse de forma efetiva. Apesar disso, tais remoções ainda assim foram maiores que as apresentadas somente para aplicação de tanino como coagulante.

\section{Investigação das hemiceluloses catiônicas-1 como auxiliar de coagulação}

A dosagem do coagulante tanino foi então fixada em $3000 \mathrm{mg} \mathrm{L}^{-1}$, dosagem mínima em que o coagulante, quando aplicado sozinho, promovia de forma eficiente o processo de coagulação em regiões ácidas, e a de hemicelulose catiônica-1 foi aumentada gradativamente de 200 até $3200 \mathrm{mg} \mathrm{L}^{-1}$, visando uma melhoria na eficiência de tratamento sem a utilização de PAC. Cabe inicialmente ressaltar que as oscilações de remoção de turbidez observadas para aplicação do tanino sozinho (3000 $\mathrm{mg} \mathrm{L}^{-1}$ ) ocorreram na região de dosagem mínima deste para o efluente em questão, considerando a elevada concentração de sólidos já mencionada.

As Figuras 7a a 7e apresentam a variação da remoção da turbidez em função do $\mathrm{pH}$ para o efluente de lavanderia industrial tratado com tanino contendo diferentes concentrações de hemiceluloses catiônicas (de 200 até $3200 \mathrm{mg} \mathrm{L}^{-1}$ ).

As faixas de atuação do tanino aplicado sozinho ou do tanino associado às hemiceluloses catiônicas-1 (quando aplicada em dosagem de até $400 \mathrm{mg} \mathrm{L}^{-1}$ ) apresentaram remoções de turbidez semelhantes para toda a faixa de $\mathrm{pH}$ investigada. Acima desta dosagem, foi verificado um aumento nas remoções de turbidez na região de pHs básicos, na qual a atuação do coagulante primário tanino era limitada. Para dosagens semelhantes entre o coagulante principal tanino e do auxiliar de coagulação hemiceluloses catiônicas-1, verificou-se remoções de turbidez superiores a $95 \%$ para toda faixa de $\mathrm{pH}$ investigada e considerando-se o alto teor de sólidos suspensos e de turbidez do efluente (maior que $1100 \mathrm{NTU}$ ). Estes resultados sugerem um efeito sinérgico entre as atuações de ambos e representam não necessidade de uma etapa de adequação de $\mathrm{pH}$ do efluente antes da etapa de coagulação. Duas possibilidades de mecanismos de atuações podem ser sugeridas: a primeira é a de que as hemiceluloses, por possuírem um pKa maior que o do tanino, num dado $\mathrm{pH}$ ela possua grupos funcionais disponíveis diferentes daqueles existentes na estrutura do tanino para o processo complementar de coagulação; a segunda é a de que as hemiceluloses estejam interagindo com tanino, evitando a formação de micelas e, portanto, tanto o coagulante quanto o auxiliar de coagulação estariam mais susceptíveis à coagulação. Porém, investigações mais detalhadas quanto à forma de aplicação de ambos os polímeros de fontes renováveis necessitam ser realizadas.

\section{Tratamento físico químico do efluente}

Após retirada de óleos e graxas (pelo abaixamento do $\mathrm{pH}$ até 2) seguida de equalização até pH 11,5, os coagulantes (tanino) e auxiliares (hemiceluloses catiônicas) foram aplicados conjuntamente nas dosagens de $3000 \mathrm{mg} \mathrm{L}^{-1}$ e $3200 \mathrm{mg} \mathrm{L}^{-1}$, respectivamente. A Tabela 4 apresenta os resultados das caracterizações analíticas dos efluentes bruto e tratado com tanino (3000 $\mathrm{mg} \mathrm{L}^{-1}$ ) e hemiceluloses catiônicas-1 (3200 $\mathrm{mg} \mathrm{L}^{-1}$ ).

Pelo perfil analítico do efluente bruto, verificou-se valores atípicos de concentrações de sólidos suspensos totais (SST) e de condutividade (sólidos totais dissolvidos), muito superiores aos encontrados por outros autores para este tipo de água residuária, sugerindo elevadas cargas de contaminantes inorgânicos também 

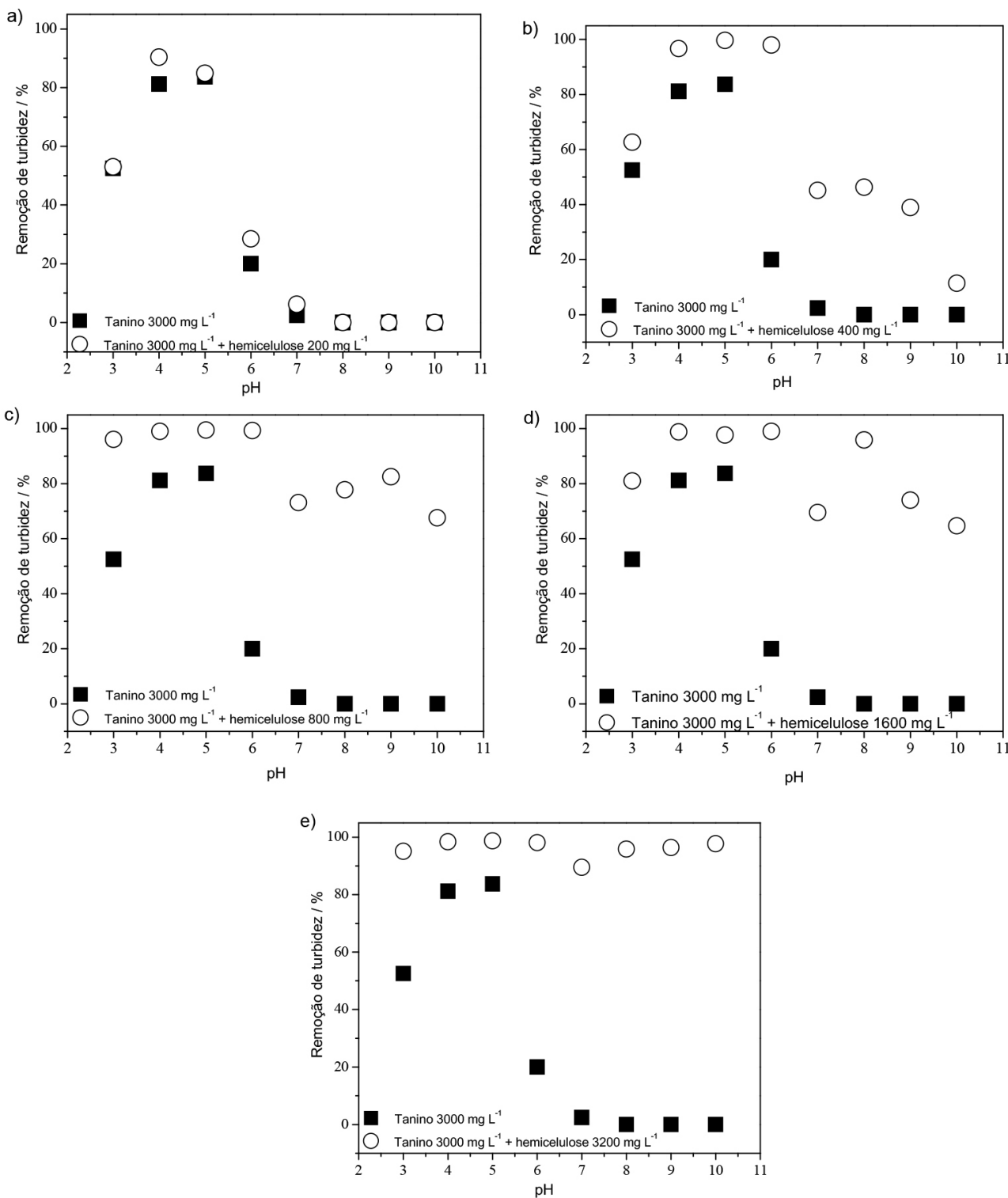

Figura 7. Variação de remoção de turbidez em função do pH para aplicação do derivado 1 (auxiliar de coagulação) no tratamento de efluente de lavanderia industrial em dosagens de: a) $200 \mathrm{mg} \mathrm{L}^{-1}$, b) $400 \mathrm{mg} \mathrm{L}^{-1}$, c) $800 \mathrm{mg} \mathrm{L}^{-1}$, d) $1600 \mathrm{mg} \mathrm{L}^{-1}$ e e) $3200 \mathrm{mg} \mathrm{L}^{-1}$

Tabela 4. Resultados das caracterizações analíticas do efluente bruto e tratado com tanino e hemiceluloses catiônicas-1

\begin{tabular}{|c|c|c|c|c|c|c|c|}
\hline Amostra & $\mathrm{pH}$ & $\begin{array}{c}\text { DQO } \\
\left(\mathrm{mg} \mathrm{L}^{-1} \mathrm{O}_{2}\right) \\
\end{array}$ & $\begin{array}{c}\text { Cor Aparente } \\
\text { (visual) }\end{array}$ & $\begin{array}{c}\text { Índice de Turbidez } \\
\text { (NTU) }\end{array}$ & $\begin{array}{c}\mathrm{SST} \\
\left(\mathrm{mg} \mathrm{L}^{-1}\right) \\
\end{array}$ & $\begin{array}{c}\text { Condutividade } \\
\left(\mu \mathrm{S} \mathrm{cm}^{-1}\right)\end{array}$ & $\begin{array}{c}\mathrm{O} \& \mathrm{G} \\
\left(\mathrm{mg} \mathrm{L}^{-1}\right)\end{array}$ \\
\hline Efluente Bruto & $\begin{array}{c}8,5^{*} \\
11,5^{* *}\end{array}$ & 81.100 & Cinza Escuro & $>1100$ & 3.250 & $>1500$ & 39.500 \\
\hline Efluente Tratado & 7 & 300 & Amarelo Claro & $20-30$ & $65-80$ & $300-400$ & 18 \\
\hline Porcentagem de Redução/\% & - & 99,63 & Sem $\operatorname{cor}^{* * *}$ & $98,2-97,3$ & $98,0-97,5$ & $80,0-73,3$ & 99,95 \\
\hline
\end{tabular}

O\&G = óleos e graxas; SST = Sólidos Suspensos Totais. *se equalizado, **sem equalizar; ***após adição de $\mathrm{H}_{2} \mathrm{O}_{2}$. O valor de Índice de Turbidez > $1100 \mathrm{NTU}$, refere-se ao LMD (limite máximo de detecção) do turbidímetro. O valor de Condutividade $>1500 \mu \mathrm{S} \mathrm{cm}^{-1}$, refere-se ao LMD (limite máximo de detecção) do condutivímetro.

confirmadas pelo elevado valor de DQO. Menezes \& Shneider ${ }^{30}$ observaram valores máximos de sólidos suspensos totais e DQO na ordem de $300 \mathrm{mg} \mathrm{L}^{-1}$ e $2.000 \mathrm{mg} \mathrm{L}^{-1}$, respectivamente, para despejos brutos das lavanderias industriais. $\mathrm{Cruz}^{28}$ menciona que, apesar dos variados tipos de diferentes substâncias encontradas na composição dos efluentes brutos, as concentrações destes agentes contaminantes são afetadas, neste caso reduzidas, pelos significativos volumes de "águas limpas" (desconcentradas) adicionadas nas várias etapas de enxágues do processo de lavagem. Como a amostragem ocorria para captar todos os processos de lavagem da lavanderia, tais valores superiores confirmam ser um efluente bruto contendo elevada sujidade.

Quanto ao efluente tratado com tanino e hemicelulose, verificaram-se porcentagens de reduções dos parâmetros analisados superiores a $98 \%$, exceto para a condutividade, esta, requerente de tratamento 
terciário seguido de filtração específica (micro ou ultrafiltração). Tais parâmetros e respectivos valores indicam a possibilidade de reuso e/ ou destinação deste efluente tratado para os corpos receptores em atendimento com as legislações ambientais vigentes (CETESB). ${ }^{31}$

\section{CONCLUSÕES}

Os resultados mostraram que foi possível preparar derivados catiônicos de hemicelulose a partir do aproveitamento da palha de milho utilizando como agente cationizante o ETA. Os derivados catiônicos produzidos são menos estáveis termicamente do que a hemicelulose natural. $\mathrm{O}$ derivado catiônico 1 apresentou um grau de substituição de 0,52 enquanto que para o derivado catiônico 2 o valor foi de 0,31 . Essa característica influencia na solubilidade em água que foi maior para o derivado catiônico 1 .

Os diagramas de coagulação mostraram que as hemiceluloses catiônicas-1 não apresentaram um comportamento que as classificassem como floculante, mas atuaram como auxiliares de coagulação ao coagulante tanino.

A atuação do tanino associado ao derivado 1 (quando aplicados em dosagem de até $200 \mathrm{mg} \mathrm{L}^{-1}$ ) forneceu remoções de turbidez semelhantes à aplicação somente do tanino, para toda a faixa de $\mathrm{pH}$ investigada. Acima desta dosagem de hemiceluloses catiônicas-1, um aumento nas remoções de turbidez na região de pHs básicos foi verificado, na qual a atuação do coagulante primário tanino era menos eficiente. Desta forma, quando o derivado 1 foi aplicado como auxiliar de coagulação (com dosagem de $3200 \mathrm{mg} \mathrm{L}^{-1}$ ), associado ao coagulante tanino, forneceram remoções de turbidez superiores a $95 \%$ para toda faixa de $\mathrm{pH}$ investigada. Considerando-se o alto teor de sólidos suspensos e de turbidez do efluente (maior que 1000 NTU), sugerem um efeito sinérgico entre as atuações de ambos, não sendo necessária uma etapa de adequação de $\mathrm{pH}$ do efluente antes da etapa de coagulação.

\section{AGRADECIMENTOS}

Os autores agradecem à FAPEMIG APQ 2279/10, CEX-APQ 0466-08 e CEX-APQ -6765-07. Ribeiro e Sousa agradecem à FAPEMIG pelas suas bolsas, CEX-APQ 0466-08 e CEX-APQ 6765-07, respectivamente. Vieira agradece à FAPEMIG pela bolsa de Doutorado. Os autores agradecem à CAPES pelo acesso ao "Portal Periódicos" e PROAP. Os autores também agradecem ao IQUFU pelos recursos para análise elementar.

\section{REFERÊNCIAS}

1. Vieira, R. G. P.; Rodrigues Filho, G.; Assunção, R. M. N.; Meireles, C. S.; Vieira, J. G.; Oliveira, G. S.; Carbohydr. Polym. 2007, 67, 182.

2. Fang, J. M.; Fowler, P.; Tomkinson, J.; Hill, C. A. S.; Carbohydr. Polym. 2002, 47, 285

3. Meireles, C. S.; Rodrigues Filho, G.; Ferreira Jr., M. F.; Cerqueira, D. A.; Assunção, R. M. N.; Ribeiro, E. A. M.; Poletto, P.; Zeni, M.; Carbohydr. Polym. 2010, 80, 954.

4. Nascimento, B.; Rodrigues Filho, G.; Frigoni, E. S.; Soares, H. M.; Meireles, C. S.; Cerqueira, D. A.; Valente, A. J. M.; Albuquerque, C. R.; Assunção, R. M. N.; Motta, L. A. C.; J. Appl. Polym. Sci. 2012, 124, 510 .
5. Biswas, S.; Huang, X.; Badger, W. R.; Nantz, M. H.; Tetrahedron Lett. 2010, 51, 1727.

6. Ren, J. L.; Liu, C. F.; Sun, R. C.; She, D.; Liu, J. C.; e-Polym. 2007a, 61, 01.

7. Seong, H. S.; Ko, S. W.; J. Soc. Dyers Colour. 1998, 114, 124.

8. Landim, A. S.; Rodrigues Filho, G.; Sousa, R. M. F.; Ribeiro, E. A. M.; Souza, F. R. B.; Vieira, J. G.; Assunção, R. M. N.; Cerqueira, D. A.; Polímeros: Ciência e Tecnologia 2013, 23, 468.

9. Sjöström, E.; Wood Chemistry. Fundamentals and Applications, Academic Press: San Diego, 1993.

10. Sun, R.; Lawther, J. M.; Banks, W. B.; Carbohydr. Polym. 1996, 29, 325.

11. Pandey, A.; Soccol, C. R.; Nigam, P.; Soccol, V. T.; Bioresour. Technol. 2000, 74, 69 .

12. Gáspár, M.; Kálmán, G.; Réczey, K.; Process Biochem. 2007, 42, 1135.

13. Silva, S. S.; Carvalho, R. R.; Fonseca, J. L. C; Garcia, R. B.; Polímeros: Ciência e Tecnologia 1998, 2, 25.

14. Fengel, D.; Wegener, G.; Wood: Chemistry ultrastructure reactions. Walter de Gruyter: Berlin, 1984.

15. Pal, S.; Mal, D.; Singh, R. P.; Carbohydr. Polym. 2005, 59, 417.

16. Haack, V.; Heinze, T.; Oelmeyer, G.; Kulicke, W. M.; Macromol. Mater. Eng. 2002, 287, 495.

17. Song, Y.; Sun, Y.; Zhang, X.; Zhou, J.; Zhang, L.; Biomacromolecules 2008, 9, 2259.

18. Souza, A. P. C.; Souza, E. A. M.; Pereira, N. C.; XX Congresso Brasileiro de Engenharia Química, Florianópolis, Brasil, 2014.

19. Nnaji, N. J. N.; Ani, J. U.; Aneke, L. E.; Onukwuli, O. D.; Okoro, U. C.; Ume, J. I.; J. Ind. Eng. Chem. 2014, 20, 1930.

20. Akter, N.; Hossain, M. A.; Hassan, M. J.; Amin, M. K.; Elias, M.; Rahman, M. M.; Asiri, A. M.; Siddiquey, I. A.; Hasnat, M. A.; J. Environ. Chem. Eng. 2016, 4, 1231.

21. Ren, J. L.; Sun, R. C.; Liu, C. F.; Lin, L.; He, C. H.; Carbohydr. Polym. 2007b, 67, 347.

22. Ren, J. L.;Sun,R. C.; Liu,C. F.; Chao, Z. Y.; Luo, W.; Polym. Degrad. Stab. 2006, 91, 2579.

23. Shen, D. K.; Gu, S; Bridgwater, A.V.; J. Anal. Appl. Pyrolysis. 2010, 87, 199.

24. Martinez, F. L.; Tese de Doutorado, Universidade do Estado do Rio de Janeiro, Brasil, 1996.

25. Coral, L. A.; Bergamasco, R.; Bassetti, F. J.; $2^{\text {nd }}$ International Workshop Advances in Cleaner Production, São Paulo, Brasil, 2009.

26. Ravina, L.; Everything you want to know about Coagulation \& Flocculation, Zeta-Meter, Inc.: Staunton, 1993.

27. Thompson Jr, J. P.; Costa, T. F. R.; Canobre, S. C.; Amaral, F. A.; Química Têxtil 2013, 65, 77.

28. Cruz, J. G. H.; Dissertação de mestrado, Universidade Federal do Rio Grande do Sul, Brasil, 2004.

29. Amaral, F. A.; Costa, T. F. R.; Thompson Jr, J. P.; Lima, D. M.; Resende, M.; Canobre, S. C.; Química Têxtil 2013, 27, 73.

30. Menezes, J. C. S. S.; Schneider, I. A. H.; Química Têxtil 2005, 80, 20.

31. CETESB; Nota técnica sobre Tecnologia de Controle-Indústria Têxtil - NT-22, 1991, 31. 